\title{
Damage Detection in Holed Carbon Fiber Composite Laminates Using Embedded Fiber Bragg Grating Sensors Based on Strain Information
}

\author{
Guoping Ding, ${ }^{1,2}$ Wenhao Song, ${ }^{3}$ Xiaoling Gao ${ }^{(D)},{ }^{3}$ and Hao Cao ${ }^{3}$ \\ ${ }^{1}$ Institute of Advanced Material and Manufacturing Technology, Wuhan University of Technology, Wuhan, China \\ ${ }^{2}$ Hubei Key Laboratory of Digital Manufacturing, Wuhan University of Technology, Wuhan, China \\ ${ }^{3}$ School of Mechanical and Electronic Engineering, Wuhan University of Technology, Wuhan, China \\ Correspondence should be addressed to Xiaoling Gao; xiaoling_whut@126.com
}

Received 26 August 2020; Revised 11 November 2020; Accepted 27 November 2020; Published 7 December 2020

Academic Editor: Jie Yang

Copyright (C) 2020 Guoping Ding et al. This is an open access article distributed under the Creative Commons Attribution License, which permits unrestricted use, distribution, and reproduction in any medium, provided the original work is properly cited.

Compared with metal materials, Carbon Fiber Reinforced Plastic (CFRP) is more excellent in performance, with high specific strength and high specific modulus. And among various CFRP structural elements, CFRP laminated plates have widespread applications. However, some indiscernible and hidden primary damage always appears on the CFRP laminated plate and will continuously evolve and expand after being loaded. This work starts with the analysis of the relationship between the changes of the strain distribution and damage expansion of CFRP laminated plates and proposes the damage recognition method of CFRP laminated plates based on strain information. Then, the CFRP laminate damage monitoring system based on Fiber Bragg Grating (FBG) sensor is established, and the CFRP laminate damage identification experiment based on FBG sensor is made to verify the accuracy of the CFRP laminate damage identification method. The results show that the maximum error between the load when the damage appears on the FBG sensor and that when the damage of CFRP laminated plates expands based on the simulated analysis does not exceed $16 \%$. The accuracy of the damage recognition method of CFRP laminated plates is verified and the damage recognition of CFRP laminated plates and their expansion process is achieved.

\section{Introduction}

Compared with metal materials, Carbon Fiber Reinforced Plastic (CFRP) has good characteristics including high specific strength, high specific modulus, fatigue resistance, and high-temperature resistance [1-4]. And among various CFRP structural elements, CFRP laminated plates are most commonly used. However, some indiscernible and hidden primary damage always appears on the CFRP laminated plates due to defective workmanship, improper transportation, and improper storage and will continuously evolve and expand after being loaded, which greatly weakens the mechanical property of CFRP laminated plates and increases the risk of accidents $[5,6]$. Besides, the damage of CFRP and that of traditional metal and other materials are distinguished in the mechanism, so some existing damage detection and monitoring methods are unsatisfactory in the usual effect. Therefore, it is very significant to recognize the damage of CFRP and their expansion. The existing common methods of the damage detection of CFRP include ultrasonic testing, radiographic testing, and acoustic emission testing [7]. Both ultrasonic testing and radiographic testing are static damage detection methods, which cannot detect the real-time dynamic of damage during the movement process of the structure. In the acoustic emission testing method, the damage is detected by a piezoelectric sensor to capture the elastic waves released when the deformation or breakage occurs in materials. However, elastic waves are easy to introduce interference and noise due to the frequency dispersion, pattern conversion, attenuation, and interface reflection during the propagation process in the composites, and the degumming failure of piezoelectric sensor and 
composites is easily caused in the connection surface so that this method is difficult to adapt to the complex working condition of CFRP. Fiber Bragg Grating (FBG) sensor is light in quality, small in volume, easy to be embedded inside composites, and easy to achieve the distributed measurement, which can, thus, online detect the components and parts of composites. After FBG sensor technology is introduced to the damage detection of composites, the shortcomings of conventional damage detection technologies including ultrasonic testing and radiographic testing can be effectively overcome. Therefore, those advantages of the FBG sensor can be used to build the FBG sensor network, and the distributed FBG sensor can be embedded inside the CFRP to online recognize its damage and expansion.

Some researchers made the following relevant works for damage detection of CFRP laminated plates based on FBG sensor. Okabe et al. embedded FBG sensor in the orthogonal laminated plate of composites, conducted static and cyclic loading experiments, and observed the spectral change of FBG sensor. Their results showed that the FBG sensor was more effective in static loading experiments and verified the applicability of the FBG sensor [8-12]. Pereira et al. embedded FBG sensor inside the composites, simulated and output the reflection spectrum shapes of FBG sensor corresponding to the composite structure under different crack conditions, and utilized the shape change of FBG sensor reflection spectrum to inspect the generation and expansion of the delamination damage and crack damage of composite structure, which theoretically verified the feasibility of this method to recognize the generation and expansion of the delamination damage and crack damage of composites [13-15]. Takeda et al. researched the detection of microscopic damage inside composites through experiments and researched the relationship between the number of matrix cracks and FBG sensor reflection spectrum through embedding FBG sensor inside the laminated composite plate. Their results showed that spectral width gradually increased with the increase in the number of matrix cracks [16]. Yashiro et al. embedded the broadband-spectrum fiber grating sensor in the CFRP composites and conducted tensile experiments for the laminated plates. The changes of spectrum recorded in their tensile experiments suggested that the spectral change of broadband-spectrum fiber grating sensor was affected by the concentrated stress and damage of laminated plates [17]. Güemes et al. conducted the damage detection by embedding FBG sensor inside composites in combination with acoustic emission technique and threepoint bending tests were performed for composite laminated plates. During their test process, the interference of other signals was effectively avoided and the behaviors of the delamination of laminated plates and the expansion of transversal cracks were recognized [18]. Yu et al. applied the FBG sensor with wide broadband and high sensitivity in composites for the acoustic emission testing, proposed a new method to recognize the types of damage in carbon fiber laminated plate, and analyzed the Lamb Waves modes of the three different characteristics measured by FBG sensor caused by transversal cracks, delamination, and fiber breakage [19].
In summary, researchers generally applied the spectral change of FBG sensor to judge damage changes of composite laminated plates or combined FBG sensor technology with acoustic emission technique to prepare a new type of FBG sensor to recognize damage. Real-time online detection is very difficult to be achieved through spectral recognition. This work embedded the FBG sensor inside the laminated plate. Based on the damage theory for composites, the realtime monitoring system for the damage of CFRP laminated plates based on FBG sensor is constructed. Through simulated analysis and experimental study, the relationship between strain and damage is detected by the FBG sensor so that the recognition of the damage of CFRP laminated plates and their expansion process is achieved.

\section{Principle of Damage Identification Based on Strain Information Using Embedded FBG Sensors}

2.1. Principle of Embedded FBG Sensors. The sensing principle of the FBG sensor is shown in Figure 1. Incident light is the broadband spectrum including multiple wavelengths. When incident to the grating region, only the optical waves with particular wavelength are reflected while other waves are not affected and directly continue to propagate along the fiber through the grating position. The wavelength of reflected waves is the Bragg wavelength:

$$
\lambda_{B}=2 n_{\mathrm{eff}} \Lambda
$$

In equation (1), $\lambda_{B}$ refers to reflect the wavelength of Fiber Bragg Grating (FBG), $\Lambda$ refers to the cycle of grating, and $n_{\text {eff }}$ refers to the effective refractive index of the grating.

According to elastic mechanics, elastooptical effect, thermal-optical effect, and the thermal expansion effect, the offset of the central wavelength of FBG can be expressed

$$
\Delta \lambda_{B}=\lambda_{B}(\alpha+\xi) \Delta \mathrm{T}+\lambda_{B}\left(1-P_{e}\right) \Delta \varepsilon .
$$

In equation (2), $\Delta \mathrm{T}$ refers to the temperature change of Fiber Bragg Grating, $\alpha$ refers to the coefficient of thermal expansion of fiber, $\xi$ refers to the thermal-optical coefficient, $\lambda_{B}$ refers to the initial wavelength value, $\Delta \varepsilon$ refers to the strain variation of Fiber Bragg Grating, $P_{e}$ refers to effective elastooptical coefficient, and the grating used in this work is quartz material with elastooptical coefficient of $P_{e}=0.22$ [9]. When the FBG sensor is in the constant temperature field,

$$
\Delta \lambda_{B}=\lambda_{B}\left(1-P_{e}\right) \Delta \varepsilon=\mathrm{K}_{\varepsilon} \varepsilon,
$$

where $\mathrm{K}_{\varepsilon}$ refers to the strain sensitivity of the grating sensor. It can be seen from equation (3) that the offset of the central wavelength of reflectance spectrum is only affected by the changes of stress field of FBG sensor in case of invariable external temperature; under the external stress field, the grating will cause the variation of grating period $\Lambda$ and refractive index $n_{\text {eff }}$, thus causing the change of FBG reflection wavelength and reflection spectrum. Therefore, corresponding stress-strain information can be gained through the detection of the change of the central wavelength of the FBG reflection spectrum. 


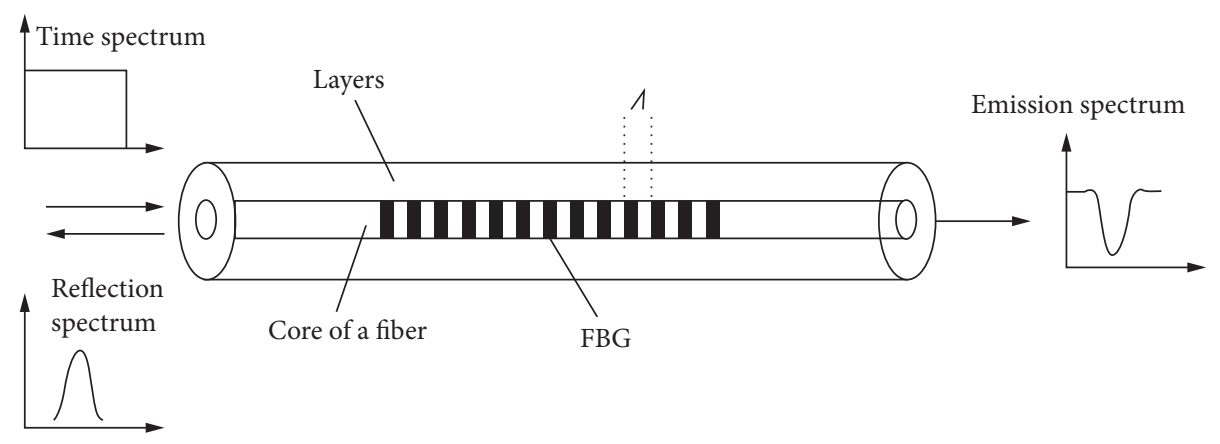

FIGURE 1: Sensing principle of FBG.

As shown in Figure 2, the wavelength-division multiplexing technology is one of significant advantages of the FBG sensor [20]. Multiple gratings are welded on fiber, and one independent and different wavelength interval is distributed in every grating within the available range of demodulator. The reflection peak of gaining every grating varies the change measured within the wavelength range. One set of demodulation equipment is applied to detect the composite spectrum formed by the reflection spectrum of all gratings. The central wavelength drift of each grating is gained within each independent wavelength interval distributed in advance to achieve the distributed measurement.

\subsection{Damage Recognition Principle of CFRP Laminated Plates} Based on FBG Sensor. When CFRP laminated plates are loaded, their damage will continuously expand with the increase of bearing load. During this damage expansion process, the change of strain among the layers of CFRP laminated plates will be very dramatic. Therefore, as shown in Figure 3, this work extracts the strain information among layers during the damage expansion process and builds the mapping relation between the strain information among the layers of CFRP laminated plates and the damage expansion degree. In this research, the FBG sensor is embedded inside the CFRP laminated plate. FBG sensor detects the strain information among layers and captures the change of the strain among lawyers to recognize the damage and their expansion, thus achieving the damage recognition method of CFRP laminated plates based on FBG sensor.

\section{Simulated Analysis for the Damage of CFRP Laminated Plates}

This study adopts the CFRP laminated plate with a length of $270 \mathrm{~mm}$, width of $31 \mathrm{~mm}$, thickness of $1.6 \mathrm{~mm}, 8$ layers, the layout way of $\left[0_{2} / 90_{2}\right] \mathrm{s}$, each thickness of $0.2 \mathrm{~mm}$, and FAW200RC38 unidirectional carbon fiber prepreg. In order to simulate the hidden original damage caused by process defects, the drilling in CFRP laminates was taken as the original prefabricated damage, where hole diameter is $1 \mathrm{~mm}$. The structure and dimension of CFRP laminated plates are shown in Figure 4.

The detailed processes of finite element simulation analysis are shown as follows:
(1) The finite element model for the tensile and load damage of CFRP laminated plates is built in ABAQUS. The structure and dimension of CFRP laminated plates are shown in Figure 4, where the structure in plane of CFRP laminated plates is the continuum shell element with the thickness of $0.19 \mathrm{~mm}$ and that among layers is interface layer element with $0.01 \mathrm{~mm}$, both of which apply offset form to generate the grid. The offset direction is set to point on the lower surface of the laminated plates to their upper surface. The generated finite element model is shown in Figure 5.

(2) Relevant material attributes and contact attributes are defined. The density, elastic parameters, and Hashin damage criterion parameters are given to the plane element of CFRP laminated plates. The layers are defined in accordance with the predetermined scheme. The element deletion is set to start when the maximum stiffness reduction of the element is 1 ; that is, when the damage variable of one element reaches 1 , this element will be deleted from the model. The interface layer element among the layers of CFRP laminated plates is assigned. The physical thickness is assigned as $0.01 \mathrm{~mm}$, the maximum stiffness reduction of the element is assigned as 1; that is, when the damage variable of one element reaches 1 , this element will be deleted from the model, and the bulk viscosity coefficient is defined as 0.00001 to improve the convergence of interface layer element.

(3) The applied tensile load and boundary conditions are determined. A reference point RP-1 was established on the right side of CFRP laminated plates. The fixed constraint is imposed on the left end of CFRP laminated plates, the rigid coupling constraint which is built between the right end of CFRP laminated plates and the reference point RP-1 is built, and the tensile displacement is imposed on this reference point as shown in Figure 6.

(4) The solutions are output. In the output manager, the stress and strain of each layer of CFRP laminated plates and the element among layers are set and output.

With the section from the edge of the hole to the right end of laminated plates as the analysis object, creating the 


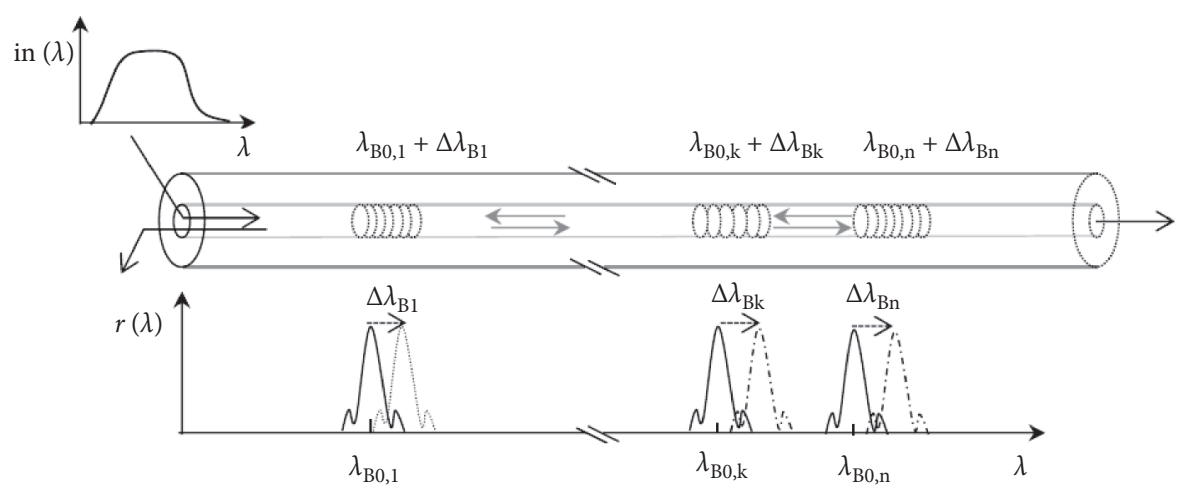

FIGURE 2: Schematic diagram of wavelength-division multiplexing technology of FBG.

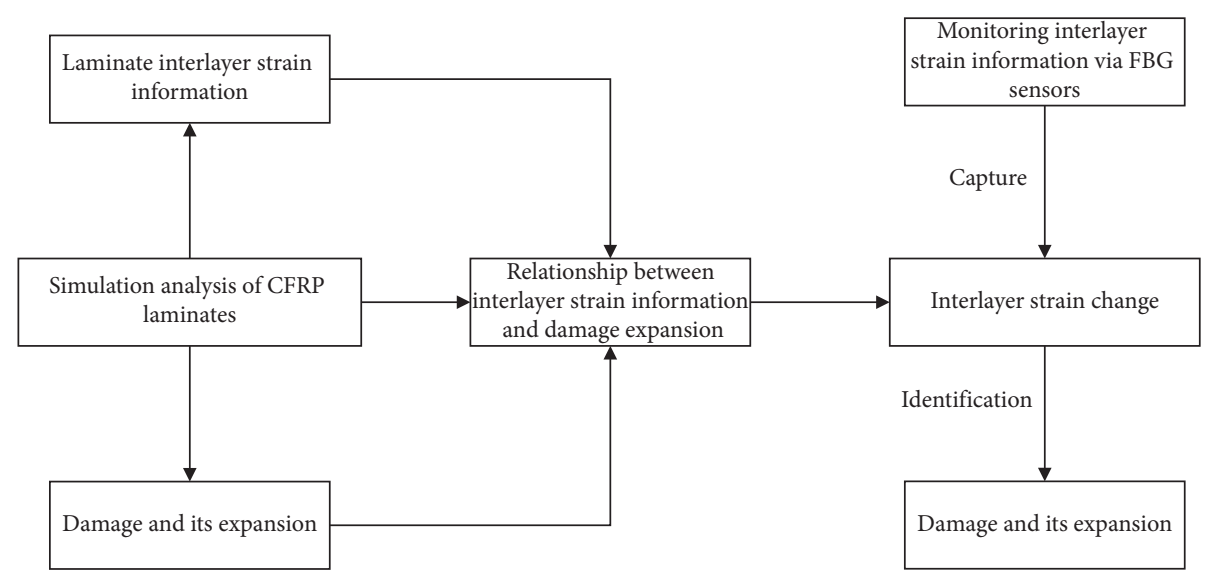

FIGURE 3: Damage recognition principle of CFRP laminated plates based on FGB sensor.

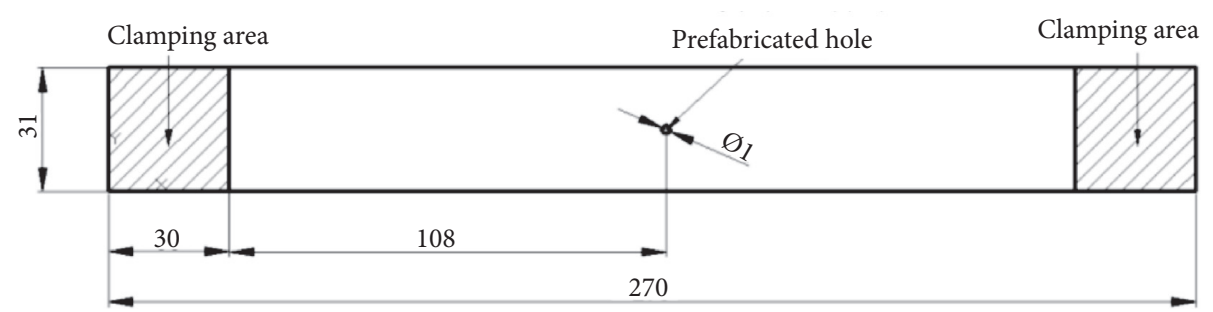

FIGURE 4: Structure and dimension of CFRP laminated plates.

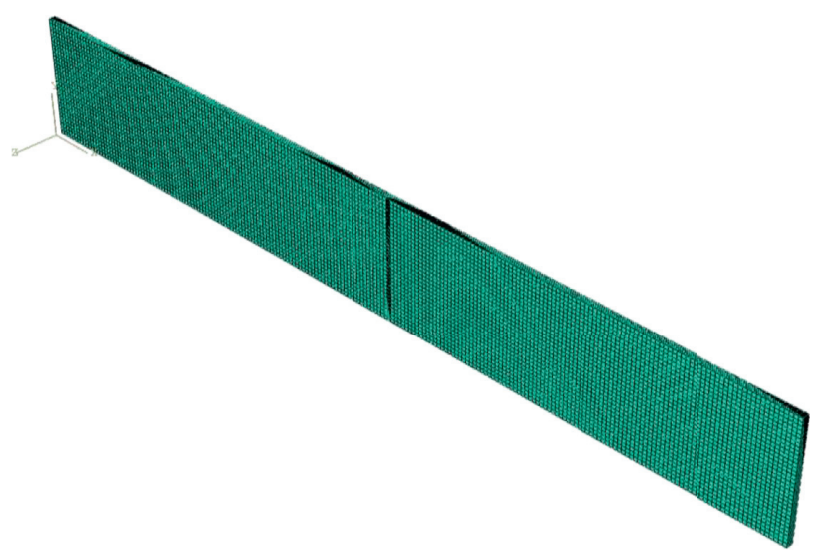

FIGURE 5: Finite element model for the tensile and load damage of CFRP laminated plates. 


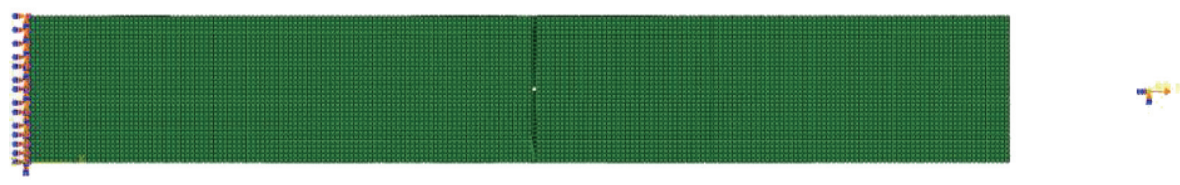

FIGURE 6: Schematic diagram of boundary conditions.

path shown in Figure 7 in the model, the strain between the first layer and the second layer of each point in the path is extracted in the simulation results and the changing situation of the strain among layers of the load is analyzed.

As shown in Figure 8(a), the tensile displacement in the end is increased from $0.3 \mathrm{~mm}$ to $1.5 \mathrm{~mm}$, and the maximum strain among layers of CFRP laminated plates appear near the hole; as tensile displacement increases, the strain among layers of each point gradually increases and the difference between maximum and minimum strains also increases.

As shown in Figure 8(b), the tensile displacement in the end increases from $1.5 \mathrm{~mm}$ to $1.6 \mathrm{~mm}$ and the strain among layers of the part near the hole produces saltation and decreases. At this time, CFRP laminated plates begin the damage expansion while the strain of the element far away from the hole continues to increase due to no damage; CFRP laminated plates restart the stress-strain distribution during the process that the end displacement increases from $1.6 \mathrm{~mm}$ to $1.8 \mathrm{~mm}$.

As shown in Figure 8(c), the strain among layers of the part near the hole does not increase with the increase of tensile displacement in the end from $1.8 \mathrm{~mm}$ to $2.1 \mathrm{~mm}$ but irregularly fluctuates, which suggests that CFRP laminated plates are redistributing the stress strain.

In summary, damage of CFRP laminated plates expands from the prefabricated damaging hole to both ends with the increase of tensile displacement of ends, causing that the strain between the first layer and the second layer of CFRP laminated plates. Therefore, damage to CFRP laminated plates can be detected according to the strain distribution changes among layers of laminated plates.

As shown in Figure 8, when damage appears near the hole of CFRP laminated plates, the strain among layers near the hole fluctuates. Such fluctuation can be regarded as the sign of damage. In order to further analyze the relationship between strain fluctuation and damage expansion, five areas (A, B, C, D, and E) are selected as shown in Figure 9, where A is nearest to the hole and $\mathrm{E}$ is farthest away from the hole. The strain-displacement curve graph is gained as shown in Figure 10 after the change of the strain among layers with the tensile displacement of ends.

(1) The simulation results show that damage appears when the displacement in the ends of CFRP laminated plates is $1.5 \mathrm{~mm}$, so the strain among layers of five areas (A, B, C, D, and E) increases linearly and does not fluctuates when the displacement is less than $1.5 \mathrm{~mm}$.

(2) When the displacement reaches $1.5 \mathrm{~mm}$, damage appears in the element near the hole, its strain rapidly decreases, and the stress-strain distribution is reperformed; the element far away from the hole has no damage, and its strain continues to increase, so the curve rate of the strain among layers of area $\mathrm{A}$ becomes negative, and its strain among layers has a decreasing trend, causing that the curve rate of the strain among layers of areas B, C, and D slightly descends.

(3) As the tensile displacement in the ends continues to increases, its damage degree becomes more and more serious and the damage range is larger and larger. Strain among layers of the part near the hole fluctuates and such fluctuation becomes smaller and smaller while that of the part without damage has no relevant changes. Therefore, the curve of strain among layers of area A firstly increases and then decreases, that of areas B, C, and D suddenly turns negative in rate and fluctuates, and that of area $\mathrm{B}$ increases all the way.

Therefore, damage of CFRP laminated plates and their expansion can be recognized according to the curve rate of the strain between the first layer and the second layer of CFRP laminated plates.

\section{Experiment}

4.1. Design and Preparation of Experimental Parts. This experiment designs and prepares two CFRP laminated plates embedded with the FBG sensor, named as testing samples A and $B$, respectively. The dimension, laying angle, number of layers, and location of prefabricated damage of two CFRP laminated plates are the same as the testing samples used in Section 3.

The basic process of preparing the test sample is as follows: pretreating the prepreg, laying the prepreg, embedding the fiber grating string, vacuum sealing, putting it into the autoclave, and maintaining the preset temperature and pressure in the tank. Time until the prepreg is completely cured, after curing, takes out the product and performs necessary postprocessing and connects the fiber grating string to the jumper and punch hole. The FBG sensor is located between the first and second layers of CFRP laminated plates, where the embedded location of the FBG sensor is shown in Figure 11. FBG5 is located in the middle of the laminate. Five FBG sensors are used in this experiment, consisting of grating string 1 (there are two gratings on fiber) and grating string 2 (there are three gratings on a fiber). The initial central wavelength distributed to the sensors FBG1, FBG2, FBG3, FBG4, and FBG5 is $1530 \mathrm{~nm}$, $1530 \mathrm{~nm}, 1540 \mathrm{~nm}, 1540 \mathrm{~nm}$, and $1550 \mathrm{~nm}$, respectively. The FAW200RC38 unidirectional carbon fiber prepreg produced 


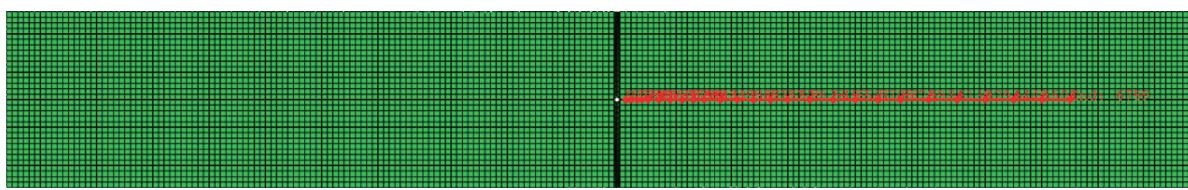

Figure 7: Pick path for the strain of CFRP laminated plates.

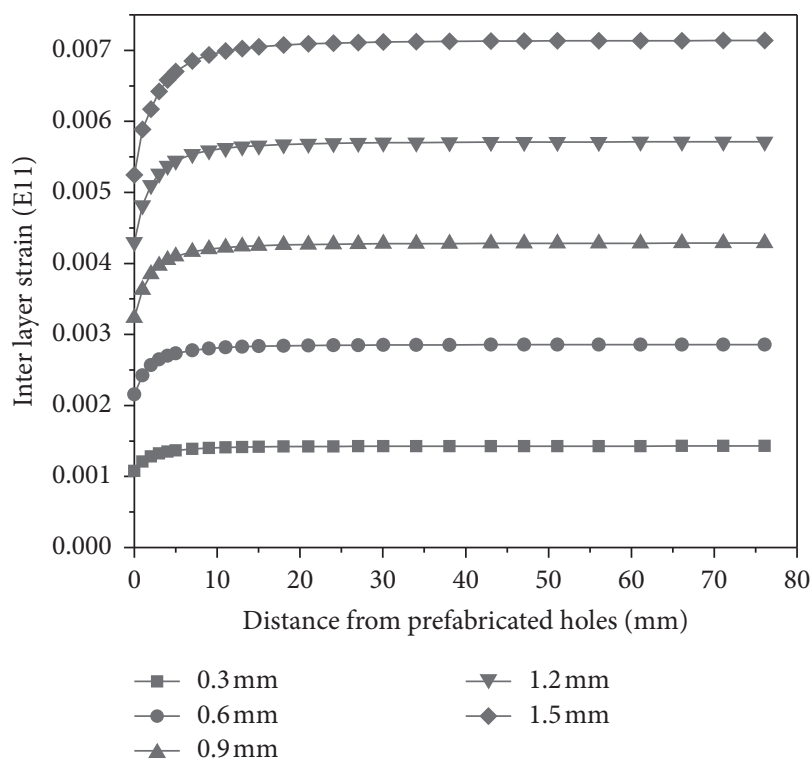

(a)

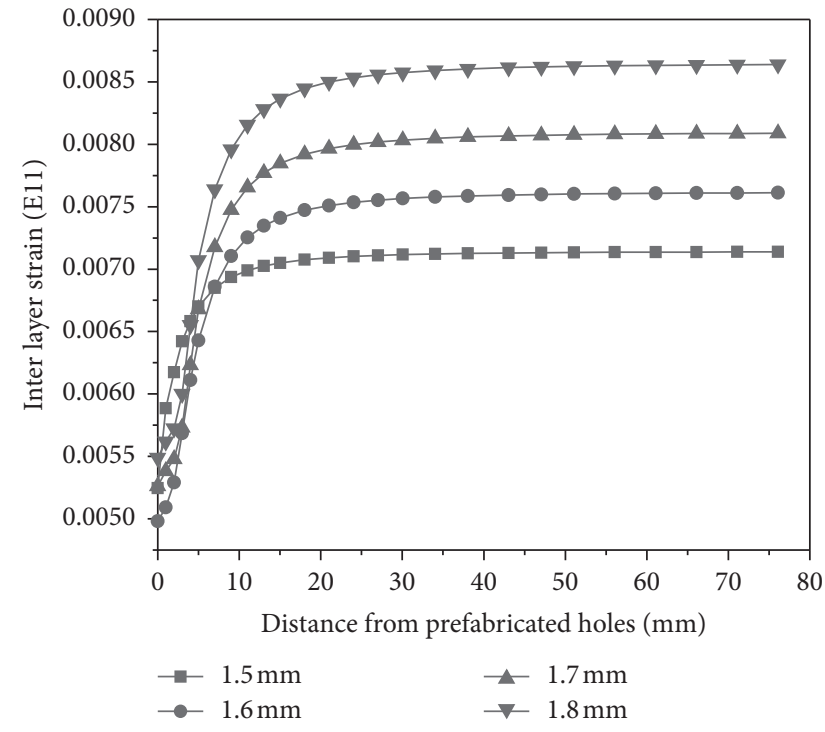

(b)

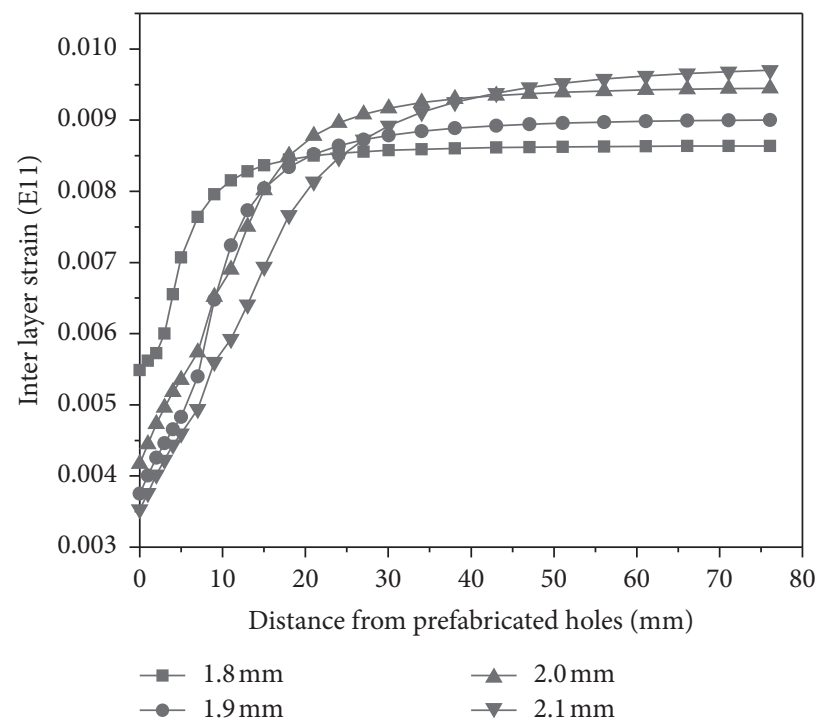

(c)

FIGURE 8: Strain among layers of CFRP laminated plates. (a) Strain among layers of CFRP laminated plates in the case of the tensile displacement of ends of $0.3 \mathrm{~mm}-1.5 \mathrm{~mm}$. (b) Strain among layers of CFRP laminated plates in the case of the tensile displacement of ends of $1.5 \mathrm{~mm}-1.8 \mathrm{~mm}$. (c) Strain among layers of CFRP laminated plates in the case of the tensile displacement of ends of $1.8 \mathrm{~mm}-2.1 \mathrm{~mm}$.

by Zhongfu Shenying Technology Co., Ltd. is adopted in the experiments, the autoclave forming process is adopted to prepare the carbon fiber/epoxy resin composite laminated plates, and the main compositions of prepreg is T700 carbon fiber and epoxy resin. According to the preset scheme, $\mathrm{BOSCH}$ electric drill is used to drill the CFRP laminated plate for prefabricated damage after the preparation of CFRP laminated plates is completed. 


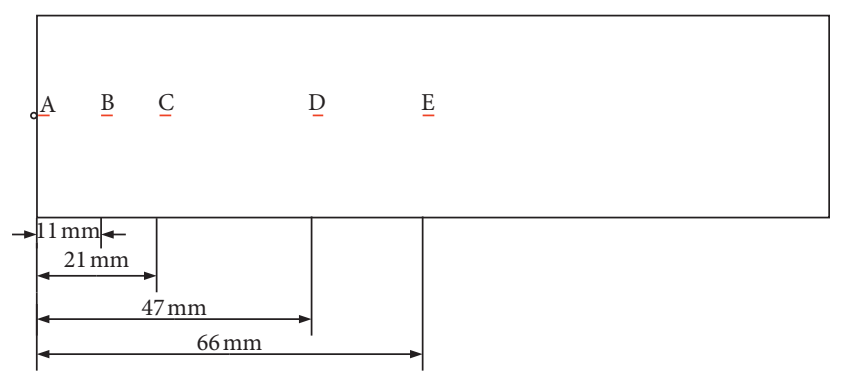

Figure 9: Five areas (A B, C, D, and E) of CFRP laminated plates.

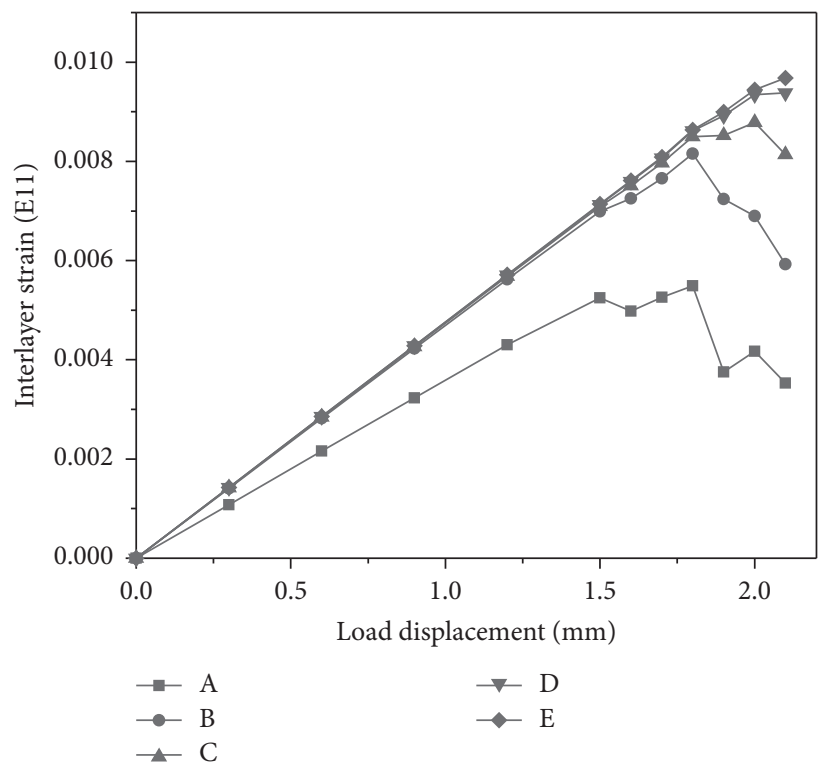

FIGURE 10: Curve graph of strain displacement.

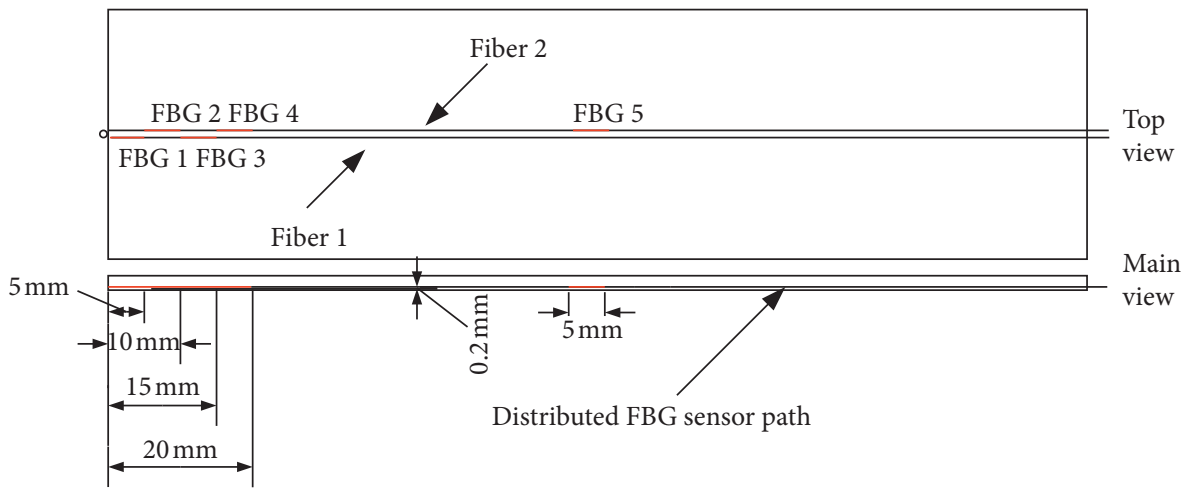

Figure 11: Embedded location of FBG sensor.

4.2. Experimental Setup. As shown in Figure 12, this experiment adopts DDL100 microprocessor-control electronic universal testing machine to load testing samples and uses the Gaussian photoelectric OPM800 demodulator to demodulate the wavelength, to gain the wavelength information of FBG sensor of testing samples A and B under same tensile load and displacement, respectively.
4.3. Experimental Procedure. DDL100 microprocessorcontrol electronic universal testing machine is adopted to load the testing samples A and B of CFRP laminated plates embedded with FBG and the load speed is set as $50 \mathrm{~N} / \mathrm{s}$. During the load process, the Gaussian photoelectric OPM800 demodulator is used to demodulate the wavelength of five FBG sensors during the load process with a frequency 


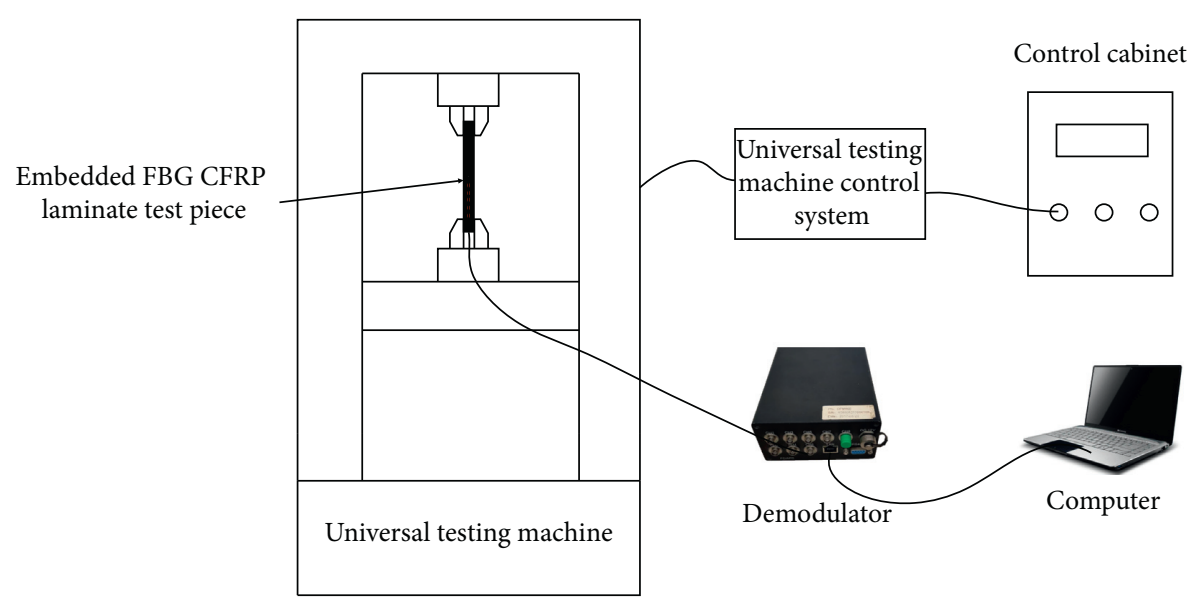

(a)

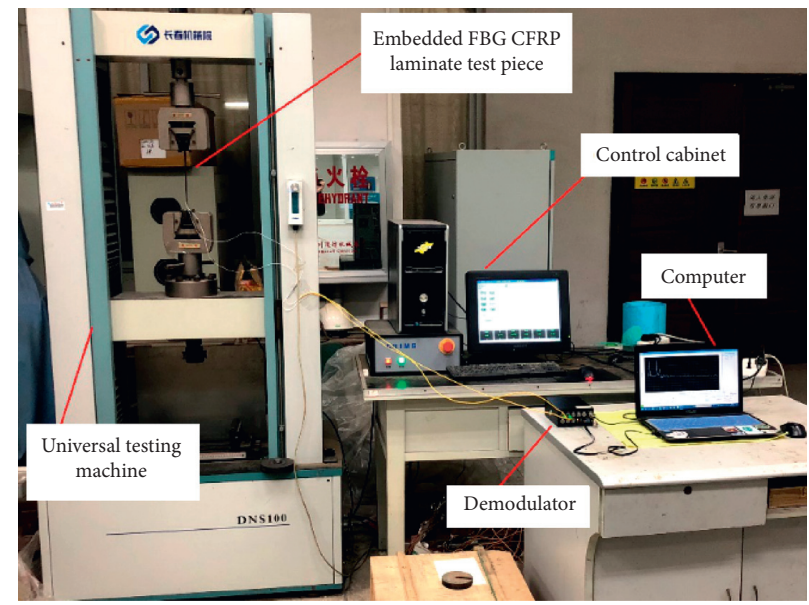

(b)

Figure 12: Experimental system. (a) Schematic diagram of the experimental system. (b) Material diagram of the experimental system.

of $1 \mathrm{~Hz}$. Information of the strain among layers of testing samples is acquired based on the wavelength information of the FBG sensor so as to recognize the damage generation and expansion process of CFRP laminated plates under the tensile load. All testing samples are loaded under the same experimental conditions.

In order to verify the experimental results, the Olympus epoch 100 phased array ultrasonic flaw detector was used to scan the embedded parts of five FBG sensors on the CFRP laminate before and after the tensile test, and the five parts were, respectively, recorded as scanning spot 1 to scanning spot 5. Ultrasonic waves are reflected on the upper surface of the probe and the CFRP laminate and displayed as beginning waves. After penetrating the CFRP laminate, they are reflected at the lower surface of the CRRP laminate and displayed as final waves. If there is no damage inside the CFRP laminate, the part between the beginning wave and the final wave is uniformly light-colored; if there is a certain degree of damage inside the CFRP laminate, the part between the beginning wave and the final wave will be messy; if the CFRP laminate has a very serious internal damage, the final wave will be difficult to identify and even disappear.
4.4. Experimental Results. Figures 13(a) and 13(b) show the change condition of the offset of FBG sensor wavelengths in embedded testing sample A and testing sample B with the time.

(1) In the initial tensile stage, the offset of the central wavelength of the FBG sensor linearly increases with the increase of tensile displacement.

(2) When the tensile load is increased to a certain value, the offset of the central wavelength of sensor FBG1 decreases after the peak value. According to previous simulation analysis, the damage appears in testing samples at this time. The obvious rattle in the experimental procedure also verifies the appearance of damage.

(3) With the continuous increase of the tensile load, the damage which appeared in the area of the sensor to FBG1 also has a certain effect on other areas. The offset of the central wavelength of sensors FBG2, FBG3, and FBG4 shows a similar change rule to the sensor FBG1 successively, which suggests that the damage also appears in the areas of FBG2, FBG3, and FBG4. 


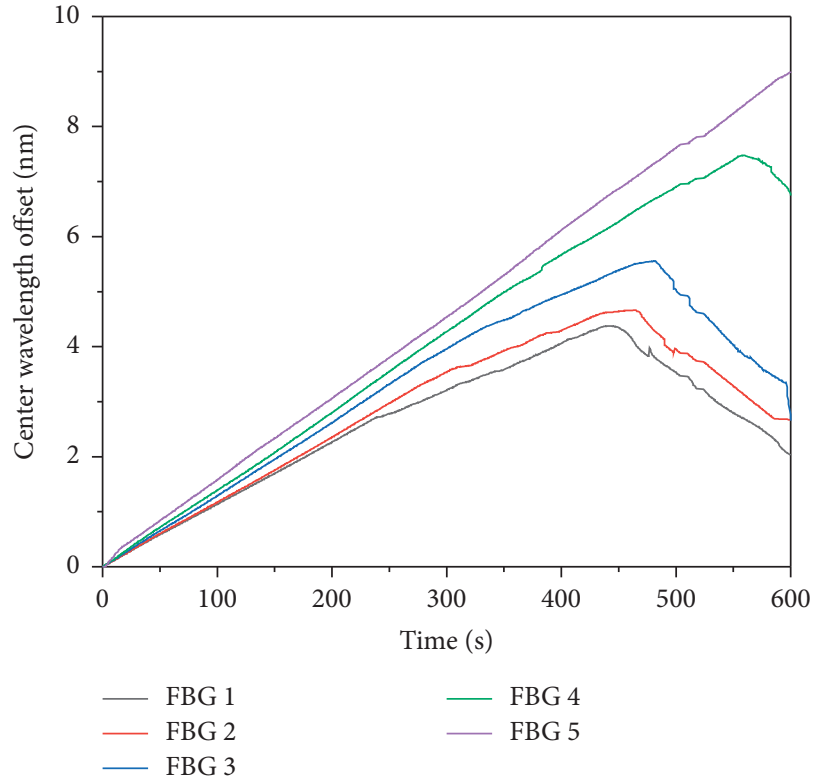

(a)

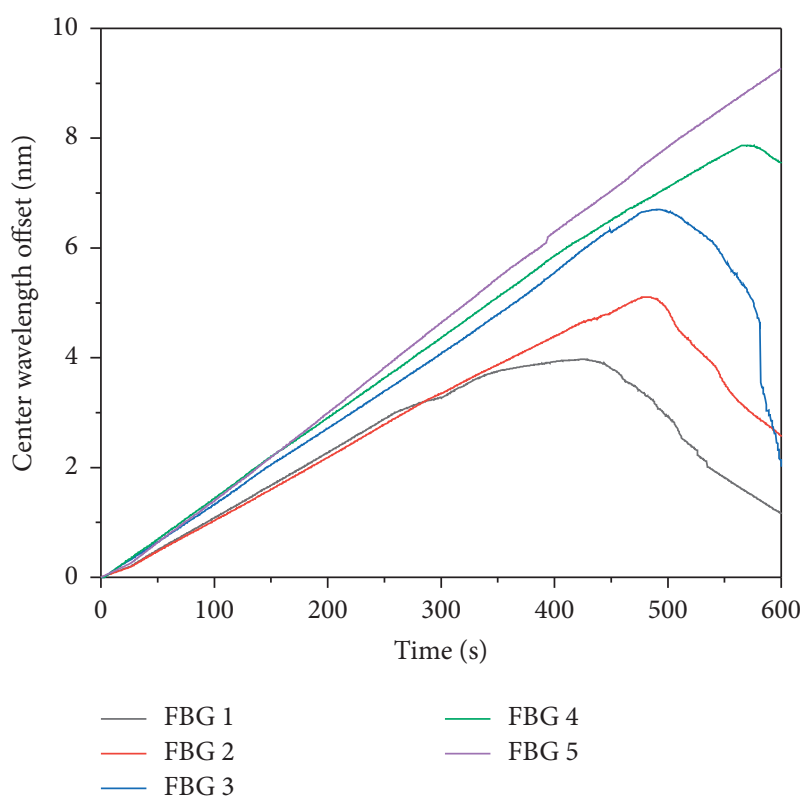

(b)

Figure 13: Curve of the offset of the central wavelength of the FBG sensor with time. (a) Measurement date of test article A. (b) Measurement date of test article B.

TABLE 1: Analysis of the experiment results of testing samples A and B.

\begin{tabular}{lccccc}
\hline & FBG1 & FBG2 & FBG3 & FBG4 & FBG5 \\
\hline Simulation value & $25267 \mathrm{~N}$ & $26766 \mathrm{~N}$ & $28491 \mathrm{~N}$ & $30179 \mathrm{~N}$ & - \\
Testing sample A & $22300 \mathrm{~N}$ & $23250 \mathrm{~N}$ & $24050 \mathrm{~N}$ & $28000 \mathrm{~N}$ & $7.2 \%$ \\
Error & $11.7 \%$ & $13.1 \%$ & $15.6 \%$ & - \\
Testing sample B & $21550 \mathrm{~N}$ & $24250 \mathrm{~N}$ & $24700 \mathrm{~N}$ & $29500 \mathrm{~N}$ & - \\
Error & $14.9 \%$ & $9.4 \%$ & $13.3 \%$ & $2.2 \%$ & - \\
\hline
\end{tabular}

(4) In the whole tensile process, the area of FBG5 has no damage and the offset of the central wavelength of sensor FBG5 increases with the increase of tensile load all the way.

In order to further verify the accuracy of the FBG sensor in detecting damage, according to the time when the grating center wavelength reaches the maximum offset, the corresponding tensile load of each FBG sensor area is extracted from the load-time curve of the universal testing machine and compared with the theoretical value. The errors are shown in Table 1, where the minimum error is $2.2 \%$ and the maximum one is $15.6 \%$.

The damage area detected by the FBG sensor and the result of the ultrasonic measurement is contrasted. Figures 14 and 15 show the results of ultrasonic scanning of testing samples before and after the tensile experiment, respectively. Before the experiment, the colors of the central area of the beginning wave and the final wave are uniform. The performance of CFRP laminated plates is good, and no damage appears. After the experiment, the end waves of the first four scanning spots become disorderly unsystematic and difficult to be recognized, the area of central area of the beginning wave and the final wave becomes smaller, the color distribution becomes uneven, and the damage appears in the scanning area of CFRP laminated plate test article. Beginning waves and final waves of scanning spot 5 before and after the experiment are hardly different, and this spot has no damage.

\section{Discussions}

(1) This work contrasts the load when the damage appears on the FBG sensor in the experimental procedure and the load when the damage of CFRP laminated plates expands by the simulated analysis. It is found that there are certain errors between experimental results and simulation results due to the influence of the errors of testing samples preparation process and experimental setup, but the maximum error does not exceed $16 \%$. This verifies the accuracy of the FBG sensor for damage detection.

(2) The damage area measured by each FBG sensor and that detected by phased array ultrasonic fault detector are compared. They have good consistency, which verifies the accuracy of the FBG sensor to detect damage area and expansion. 


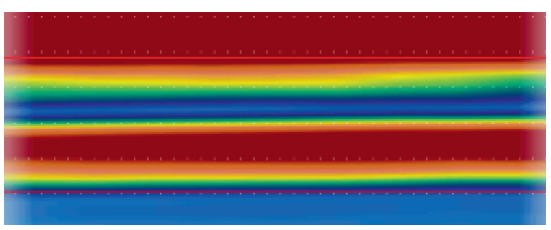

(a)

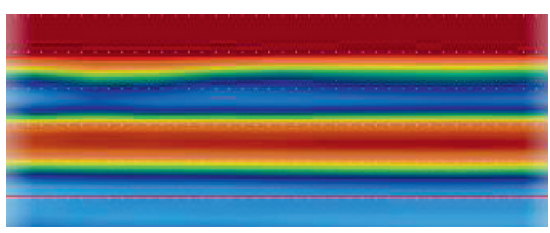

(d)

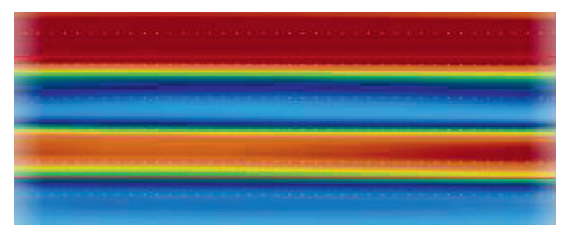

(b)

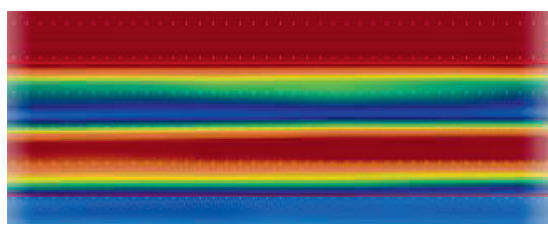

(c)

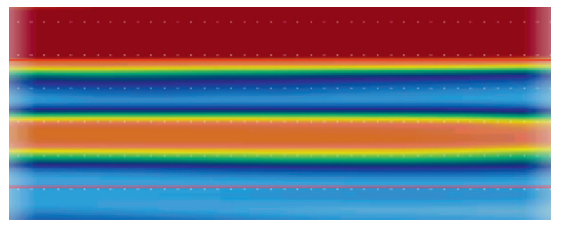

(e)

FIGURE 14: Results of ultrasonic scanning of testing samples before tensile experiments. (a) Scanning spot 1. (b) Scanning spot 2. (c) Scanning spot 3. (d) Scanning spot 4. (e) Scanning spot 5.

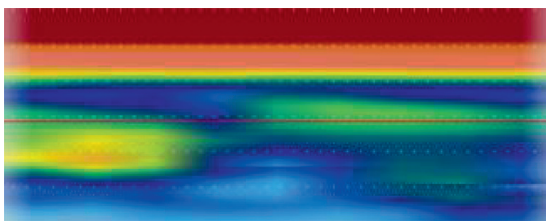

(a)

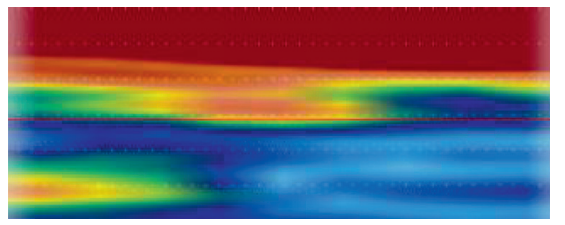

(d)

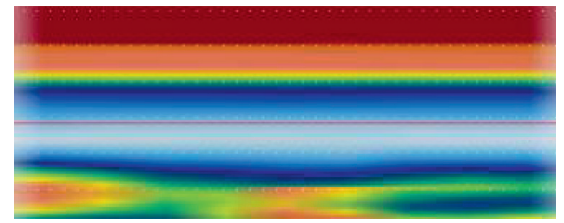

(b)

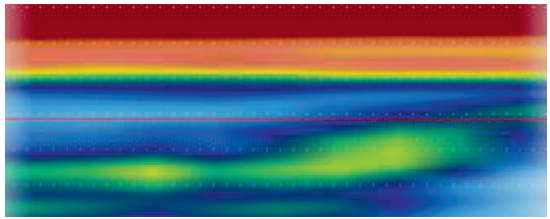

(c)

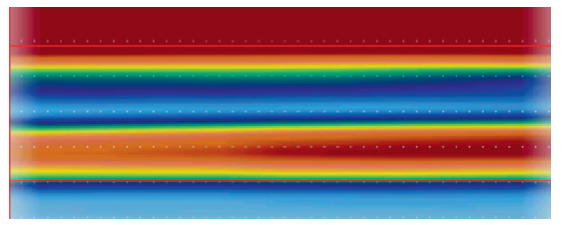

(e)

FIGURE 15: Results of ultrasonic scanning of testing samples after tensile experiments. (a) Scanning spot 1. (b) Scanning spot 2. (c) Scanning spot 3. (d) Scanning spot 4. (e) Scanning spot 5.

\section{Conclusions}

In this work, a suitable failure criterion is firstly selected in ABAQUS for the simulation analysis of the initial damage and their damage of CFRP laminated plates, which provide a foundation for the damage recognition method of CFRP laminated plates based on strain information. Then, the mapping relation between the changes of strain distribution between the first layer and the second layer of CFRP laminated plates and the damage expansion evolution is gained based on the results of simulation analysis and the damage recognition method of CFRP laminated plates is built. Finally, with distributed FBG sensor as the key component, the damage monitoring system of CFRP laminated plates based on FBG sensor is built and the sensor recognition experiment of CFRP laminated plates based on FBG sensor is made, which verifies the reliability of the damage recognition method of CFRP laminated plates. The experimental results and simulation results have good consistency, and the maximum error does not exceed $16 \%$, which verify the accuracy of the FBG sensor for damage detection. The damage area measured by each FBG sensor and that detected by phased array ultrasonic fault detector are compared, which verifies the accuracy of FBG sensor to detect damage area and expansion.

\section{Data Availability}

All data and models generated or used during the study appear in the article.

\section{Conflicts of Interest}

The authors declare that they have no conflicts of interest.

\section{Acknowledgments}

This study was supported by National Natural Science Foundation of China (NSFC) (51775400).

\section{References}

[1] L. Liu, C. Jia, J. He et al., "Interfacial characterization, control and modification of carbon fiber reinforced polymer composites," Composites Science and Technology, vol. 121, pp. 56-72, 2015. 
[2] S. Bowman, Modification of Carbon Fiber Sizing Adapted to the Use of Thermoplastic Towpregs and Their Composite Materials, Donghua University, Shanghai, China, 2018.

[3] F. F. Wang, "Study on the application of carbon fiber composite materials in high-speed trains," Materials Science Forum, vol. 893, pp. 31-34, 2017.

[4] M. Gong, L. Sun, S. G. Sun, A.-Q. Tian, and Q. Li, "Simulation and experimentation of wide frequency electromagnetic shielding coating used for carbon fiber composite materials of track vehicles," International Journal of Modern Physics B, vol. 33, no. 1n03, Article ID 1940020, 2018.

[5] M. S. Aravinda Kumar, S. Panda, and D. Chakraborty, "Design and analysis of a smart graded fiber-reinforced composite laminated plate," Composite Structures, vol. 124, 2015.

[6] A. Kumar, S. Panda, S. Kumar, and D. Chakraborty, "A design of laminated composite plates using graded orthotropic fiberreinforced composite plies," Composites Part B: Engineering, vol. 79, pp. 476-493, 2015.

[7] H. Fernandes, H. Zhang, A. Figueiredo, C. Ibarra-Castanedo, G. Guimarares, and X. Maldague, "Carbon fiber composite inspection and defect characterization using active infrared thermography: numerical simulations and experimental results," Applied Optics, vol. 55, no. 34, pp. D46-D53, 2016.

[8] T. Okabe and S. Yashiro, "Damage detection in holed composite laminates using an embedded FBG sensor," Composites Part A: Applied Science and Manufacturing, vol. 43, no. 3, pp. 388-397, 2012.

[9] M. Marcelo, R. C. S. B. Allil, A. Bessie, and F. V. B. De Naza, "A guide to fiber Bragg grating sensors," Current Trends in Short-and Long-Period Fiber Gratings, 2013.

[10] R. Manjusha, R. Ginu, S. Yuliya, and G. Farrell, "Overview of fiber optic sensor technologies for strain/temperature sensing applications in composite materials," Sensors, vol. 16, no. 1, p. 99, 2016.

[11] D. Kinet, P. Mégret, K. Goossen, L. Qiu, D. Heider, and C. Caucheteur, "Fiber Bragg grating sensors toward structural health monitoring in composite materials: challenges and solutions," Sensors, vol. 14, no. 4, pp. 7394-7419, 2014.

[12] T. Okabe, M. Nishikawa, and N. Takeda, "Numerical modeling of progressive damage in fiber reinforced plastic crossply laminates," Composites Science and Technology, vol. 68, no. 10-11, pp. 2282-2289, 2008.

[13] G. F. Pereira, L. P. Mikkelsen, and M. Mcgugan, "Crack detection in fibre reinforced plastic structures using embedded fibre Bragg grating sensors: theory, model development and experimental validation," PLoS One, vol. 10, no. 10, Article ID e0141495, 2015.

[14] G. Pereira, L. Mikkelsen, and M. Mcgugan, "Crack growth monitoring by embedded optical Fibre Bragg Grating sensors: fibre reinforced plastic crack growing detection," in Proceedings of the 3rd International Conference on Photonics, Optics and Laser Technology, Berlin, Germany, Mar 2015.

[15] G. Pereira, L. P. Mikkelsen, and M. Mcgugan, "Embedded fibre bragg grating sensor response model: crack growing detection in fibre reinforced plastic materials," in Proceedings of the 11th International Conference on Damage Assessment of Structures (DAMAS 2015), Ghent, Belgium, August 2015.

[16] S.-I. Takeda, T. Yokozeki, and T. Ogasawara, "Damage monitoring of polymer-lined carbon fibre-reinforced plastic using small-diameter fibre Bragg grating sensors," Journal of Reinforced Plastics and Composites, vol. 34, no. 6, pp. 454-462, 2015.
[17] S. Yashiro, T. Okabe, N. Toyama, and N. Takeda, "Monitoring damage in holed CFRP laminates using embedded chirped FBG sensors," International Journal of Solids and Structures, vol. 44, no. 2, pp. 603-613, 2007.

[18] A. Güemes, A. Fernandez-Lopez, and B. Hernandez-Crespo, "Monitoring damage growth in composite materials by FBG sensors," in Proceedings of the 5th International Symposium on Ndt in Aerospace, Singapore, November 2013.

[19] F.-M. Yu, Y. Okabe, Q. Wu, and N. Shigeta, "A novel method of identifying damage types in carbon fiber-reinforced plastic cross-ply laminates based on acoustic emission detection using a fiber-optic sensor," Composites Science and Technology, vol. 135, pp. 116-122, 2016.

[20] C.-H. Yeh, Y.-H. Zhuang, N. Tsai, and C.-W. Chow, "Capacity and capability enhancements of FBG sensor system by utilizing intensity and WDM detection technique," Smart Materials and Structures, vol. 26, no. 3, Article ID 35026, 2017. 